are equivalent to Einstein's equation in vacuum with a cosmological constant,

$$
G^{\mu \nu}+\lambda g^{\mu v}=0
$$

when $\lambda=6 \mathrm{~A}$.

Accordingly eqs. (3), (4) and (14), with the algebraic equations (23), give a full deseription of Einstein's equations of gravitation.

Full details of these results with proofs, will be published elsewhere.

\title{
On the Renormalization Magnon Energies in an Ordered Ferromagnetic Alloy.
}

\author{
F. LEONI \\ Laboratorio di Fisica Nucleare Applicata \\ Centro studi Nucleari della Casaccia del CNEN - Roma \\ (Lett. Nuoro Cimento. 3, $592(1970)$ )
}

Please read eq. (3) of this paper as follows:

(3)

$$
\begin{aligned}
E_{k \lambda}=\omega_{k \lambda}+2 \sum_{q} c_{\substack{k q \\
\lambda \lambda}} n_{q \lambda}-2 \sum_{\substack{q \lambda^{\prime} \\
\left(\hat{\lambda} \neq \lambda^{\prime}\right)}} c_{k \lambda^{\prime}} n_{q \lambda^{\prime}}= \\
=\Delta_{\lambda}+2 g_{\lambda \lambda} \sum_{q} n_{q \lambda}+2 \sum_{\lambda^{\prime} q} g_{\lambda \lambda^{\prime}} n_{q \lambda^{\prime}}+\gamma_{\lambda \lambda} \sum_{q} q^{2} n_{q \lambda}+ \\
+k^{2}\left[D_{\lambda}+2 f_{\lambda \lambda} \sum_{q} q^{2} n_{q \lambda}+2 \sum_{\lambda^{\prime}} f_{\lambda \lambda^{\prime}} \sum_{q} q^{2} n_{q \lambda^{\prime}}+\sum_{\lambda^{\prime} q} \gamma_{\lambda \lambda^{\prime}} n_{q \lambda}\right]
\end{aligned}
$$

(C) by Società Italiana di Fisica 